\title{
Energy Load Balancing for Fixed Clustering in Wireless Sensor Networks
}

\author{
Syed Amjad Ali \\ Department of Computer Technology and Information Systems \\ Bilkent University \\ Ankara, Turkey \\ Email: syedali@bilkent.edu.tr
}

\author{
Cuneyt Sevgi \\ Department of Information Technology \\ Isik University \\ Istanbul, Turkey \\ Email: csevgi@isikun.edu.tr
}

\begin{abstract}
Clustering can be used as an effective technique to achieve both energy load balancing and an extended lifetime for a wireless sensor network (WSN). This paper presents a novel approach that first creates energy balanced fixed/static clusters, and then, to attain energy load balancing within each fixed cluster, rotates the role of cluster head through uniformly quantized energy levels based approach to prolong the overall network lifetime. The method provided herein, not only provides near-dynamic clustering performance but also reduces the complexity due to the fact that cluster formation phase is implemented once. The presented simulation results clearly show the efficacy of this proposed algorithm and thus, it can be used as a practical approach to obtain maximized network lifetime for energy balanced clusters in fixed clustering environments.
\end{abstract}

Keywords-Energy balanced fixed clustering; energy load balancing; network lifetime; uniform quantization; wireless sensor network.

\section{INTRODUCTION}

A Wireless sensor network (WSN) [1] usually contains a large number of MEMS-based sensor nodes with limited processing, memory, radio communication and energy resources. Due to these limited resources, monitoring the interested physical phenomenon by numerous sensing devices for an extended period of time, makes it a complex and challenging task. Since, sensor nodes are usually battery operated, studying and analyzing the lifetime of WSNs are of vital importance as the lifetime needs to be maximized.

Clustering is used commonly as a basic and effective approach to design energy efficient sensor networks with extended lifetime as it reduces the communication overhead by decreasing the energy consumption due to shorter communication distance. There exist extensive research work that uses different types of clustering techniques [2], [3] to prolong a network lifetime by minimizing the energy usage and applying energy load balancing via rotating the role of cluster head within each cluster. The classification of these clustering methods can be carried out based on different perspectives, for instance, there are fixed/static or dynamic clustering algorithms that may use a centralized, distributed or hybrid algorithm to form clusters.

In centralized clustering algorithms, the base station
(BS) takes the leading role in selecting the cluster heads. On the other hand, in distributed clustering algorithms, every sensor determines its role based on certain system parameters such as residual energy or some probabilistic value. Protocols that utilize hybrid approach carry out their cluster formation and maintenance task with the help of both the BS and the sensor nodes. Here, static and centralized clustering will be of our main interest.

The Low-Energy Adaptive Clustering Hierarchy (LEACH) [4], [5] protocol is a simple and elegant approach to create dynamic clusters using a distributed algorithm that rotates cluster heads by assigning a probabilistic indicator function to each node in the network. In [5], a simulated annealing based centralized version of LEACH called LEACH-C is described to improve the lifetime of a network by ensuring that the network always has a certain number of clusters in the network as the standard LEACH algorithm does not guarantee to have the same number of cluster heads in each round due to its probabilistic nature. The Hybrid Energy-Efficient Distributed (HEED) [6] algorithm considers quasi-stationary sensor network and chooses cluster heads based on a probabilistically scaled residual energy model and routes the sensed data to the BS using multi-hop communication.

Authors in [5] also proposed a fixed clustering counterpart of LEACH-C, named, LEACH-F to provide a comparison between fixed and dynamic clustering approaches. In [7], a fixed clustering hierarchy arithmetic based on energy prediction (FCHEP) method is introduced to solve energy efficiency problem by predicting the residual energy of nodes and appointing an automatic cluster head having the highest remaining energy. Similarly, a different residual energy based scheme for fixed clustering called EnergyEfficient Protocol with Static Clustering (EEPSC) [8] is introduced for better network lifetime performance by again taking use of signal intensity. An enhancement to EEPSC is achieved by additionally considering spatial distribution of sensors to reduce intra-cluster communication as suggested in [9]. A hybrid approach using both fixed and static clustering called Dynamic/Static Clustering protocol (DSC) is proposed in [10] by extending LEACH-C.

Although, all of the above mentioned protocols have 
their advantages and shortcomings they generally do not highlight explicitly how to handle energy load balancing problem in WSNs so that the lifetime of the network can be extended and also the gap between the death of the first and the last node can easily be minimized. This paper focuses on the formation of optimum number of fixed clusters in a randomly deployed WSN consisting of stationary nodes. Moreover, this work also addresses the issues related not only to maximize the network lifetime but also to achieve energy load balancing. The novelty of this work is that it introduces a new centralized two-phased protocol to first obtain energy balanced clusters and then, to enable the sensor nodes within each fixed cluster share their residual energy information as part of their transmitted packets to the cluster head in the form of a uniformly quantized energy level as suggested in [11]. This sharing of quantized energy information enables us to reach near optimum energy load balancing which leads to a prolonged system lifetime.

The remaining sections of this paper are organized in the following order: Section II and its subsections provide details about the used network model, the energy model of the system and the definition of the lifetime of the WSN and provide two different heuristic lifetime curves to highlight the problem at hand. In Section III, the proposed two-phased scheme for energy balanced fixed clustering and the rotation of cluster head in each cluster using uniformly quantized energy levels based algorithm are explained in detail. Section IV focuses on the performance analysis of proposed scheme. Finally, Section V concludes the paper by summarizing the findings of the work.

\section{SySTEM MODEL}

\section{A. Network Model}

The sensor network model used in this paper is illustrated graphically in Fig. 1. We consider a randomly deployed WSN consisting of two distinct "roles" of nodes: cluster heads and regular sensor nodes. Each node is aware of its position and residual energy and is equipped with a limited initial energy supply and has moderate processing power and data transmission capabilities. There exist a fixed number of clusters in the network for each round of communication and only one cluster head in each cluster. A sensor can only transmit its data to an associated cluster head which forwards the aggregated data as a single packet to the BS. The association between the sensors and their corresponding cluster head in each cluster is determined by energy balanced fixed clustering algorithm as explained later.

\section{B. Energy Model}

The energy consumption model proposed in [5] is used here as well. Thus, to transmit a $p$-bit message to a distance $d$, the energy cost of transmitter can be expressed as

$$
E_{T x}(p, d)=\left\{\begin{array}{cc}
p\left(E_{\text {elec }}+\epsilon_{f s} d^{2}\right), & d<d_{0} \\
p\left(E_{\text {elec }}+\epsilon_{m p} d^{4}\right), & d \geq d_{0}
\end{array}\right.
$$

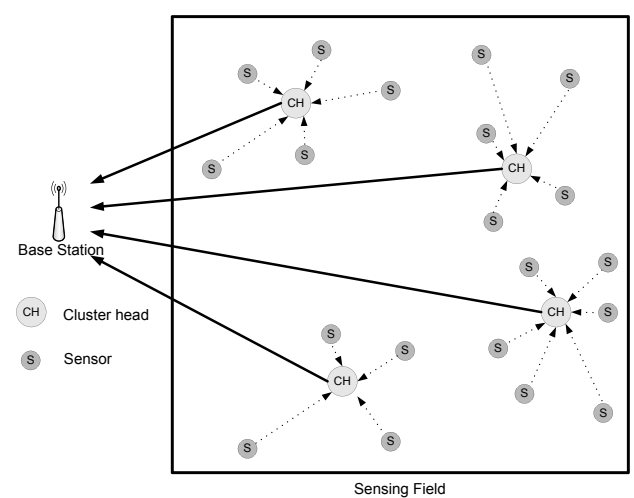

Figure 1. Wireless sensor network model.

here $E_{\text {elec }}$ is the constant amount of energy consumed by the electronics, $\epsilon_{f s}$ is the power loss of free space and $\epsilon_{m p}$ is power loss due to multipath fading. To receive a $p$-bit message the receiver spends $E_{R x}(p)=p E_{\text {elec }}$ amount of energy.

\section{Heuristic Lifetime Curves}

In a WSN application, the lifetime of the network is usually defined as either the amount of time elapsed until the first node completely depletes its energy or the last node in the network dies. In our work, we consider the former case and measure the network lifetime in terms of the number of rounds of periodical data transmission. We believe that once a node dies, the network will become unstable and will not provide the necessary coverage. The behavior of the lifetime curve of a WSN is mainly determined by how evenly the load is balanced in terms of available energy of the the nodes. It is obvious that cluster heads spend more energy than the ordinary sensor nodes as they consume energy for sensing the environment, receiving data from the associated nodes, aggregating the data and forwarding it to the BS. Therefore, it is important to rotate the role of cluster heads in an optimized manner in order not to over utilize any sensor node and to distribute the load evenly among all the sensors.

Fig. 2 heuristically shows two different lifetime performance curves with different energy load balancing characteristics. In an ideal energy load balancing case, the workload is evenly distributed such that the energy dissipated by each node is balanced and the entire network dies out at the same time. Although, it is an ideal model and is difficult to achieve in practical scenarios, it will serve as a good reference point to assess the performance of the proposed model. The poor energy load balancing case shows that some nodes are overly utilized as compared to others and thus, the time elapsed between the exhaustion of the first and the last node is moderately high, i.e., a curve with a longer tail. 


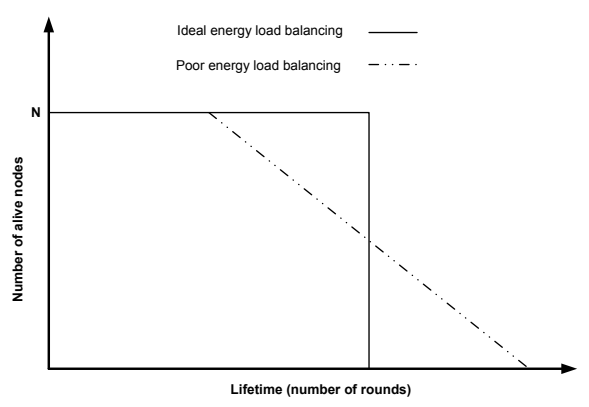

Figure 2. Heuristic lifetime curves.

\section{ENERGy BALANCEd FiXed Clustering (EBFC) WITH LOAD BALANCING}

In order to achieve energy load balancing in fixed clustering a two-phase approach is suggested. The need for this new algorithm is due to the fact that most existing clustering algorithms fail to provide near optimum load balancing. In Phase I, a new algorithm to create energy balanced clusters is presented followed by Phase II where energy load balancing within each cluster is achieved by rotating the role of cluster head in a cluster. The details regarding the suggested approach are provided in following subsections.

\section{A. Phase I: Energy Balanced Fixed Clustering (EBFC)}

For EBFC, we propose a new one time cluster formation algorithm after the random deployment of sensor nodes over a sensing field as shown in Fig. 3 using the following steps:

1) All nodes uniformly quantize their energy levels based on the required number of quantization levels by using the given relationship

$$
E_{\max }=K \Delta+E_{t h}, \quad \Delta=a_{i+1}-a_{i} .
$$

here, $K$ is the required number of quantization levels, $\Delta$ represents the equally spaced energy intervals and $E_{t h}$ is the minimum threshold energy required to directly transmit a packet from the furthest node in the network to the BS (pre-configured before the deployment). The disjoint and equally-spaced energy level regions can be expressed as $R_{1}=\left[E_{t h}, a_{1}\right]$, $R_{2}=\left(a_{1}, a_{2}\right], \ldots . ., R_{K}=\left(a_{K-1}, E_{\max }\right]$. Hence, the number of bits required to represent the discretized residual energy of each node becomes $q=\log (K)$.

2) Each node sends its location information to the BS.

3) The BS forms $n$ initial clusters using conventional $k$-means clustering algorithm. Once $n$ clusters are created, it is assumed that all nodes in each cluster are located at the centroid of each cluster i.e., intra-cluster communication is ignored.

4) Label each cluster in ascending order i.e., the cluster closest to the BS (using Euclidian distance) as cluster 1 and the second closest as cluster 2 and so on.
5) Compute the total number of packets $X_{i}$ that a single node can transmit to the BS from the centroid of each cluster (straightforward computation as the distance to BS from the centroid and the initial energy of the node are known).

6) The following relationship for the total number of packets $X_{i}$ that can be transmitted by a single node located at the centroid $C_{i}$ to the BS holds

$$
X_{1} \geq X_{2} \geq \cdots \geq X_{n-1} \geq X_{n}
$$

as $d_{1} \leq d_{2} \leq \cdots \leq d_{n-1} \leq d_{n}$. The above relationship simply means that a node that is closest to the BS will send more packets to the BS than those that are further from the BS.

7) In order to form energy balanced clusters, we need to make sure that each fixed cluster should be able to send an equal number of packets to the BS. To achieve this, the total number of nodes in distant clusters must be greater than the ones that are closer to the BS. Hence, the following ratios can be used to provide us with an estimate about the number of packets that can be send to the BS

$$
\frac{X_{1}}{X_{1}}, \frac{X_{1}}{X_{2}}, \cdots, \frac{X_{1}}{X_{n-1}}, \frac{X_{1}}{X_{n}}
$$

The above relationship can be interpreted as, if a node in cluster $_{1}$ requires one unit of energy to send $X_{1}$ number of packets to the BS, cluster ${ }_{2}$, cluster $_{3}$ and so on will require $X_{1} / X_{i}$ units of energy to send the same number of packets to the BS.

8) Since there are $N$ number of deployed nodes in the system, we have $N$ units of available total energy and the following relationship between the number of nodes in each disjoint cluster $N_{i}$ and the total deployed nodes $N$ holds

$$
N_{1}+N_{2}+\cdots+N_{n-1}+N_{n}=N
$$

The above equation can written alternatively by using the sum of the scaled ratios $X_{1} / X_{i}$ as

$$
\beta X_{1}\left(\frac{1}{X_{1}}+\frac{1}{X_{2}}+\cdots+\frac{1}{X_{n-1}}+\frac{1}{X_{n}}\right)=N
$$

Here, the multiplication factor $\beta$ will determine the number of nodes that should belong to a cluster in order to form energy balanced clusters. In the above equation, since all parameters are known $\beta$ can be computed easily.

9) Multiplying $\beta$ with the ratios given in step 7 and ceiling the value will give us the total number of nodes that are required in each cluster $\left(N_{i}\right.$ 's) to obtain energy balanced clusters.

10) Since initial $k$-means clustering will contain either extra or less number of nodes than the ones required due to step 9, each cluster will have to either borrow 


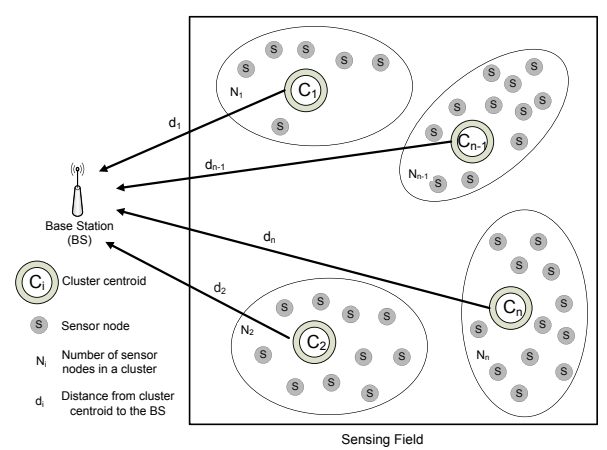

Figure 3. Energy balanced fixed clusters.

or give away nodes to other clusters to make sure that energy balanced clusters with required number of nodes in each cluster $N_{i}$ are created. In order to obtain energy balanced clusters, the clusters with extra nodes will allow clusters with missing nodes (deficient clusters) to borrow the missing number of nodes using the minimum Euclidean distance criteria i.e., a deficient cluster will borrow any node from extra nodes clusters that will be closest to its centroid in term of Euclidean distance.

11) After borrowing and lending away nodes the new centroid value of each cluster will change and it needs to be calculated iteratively until the system reaches an unchanging state i.e., a state is reached when the new centroid does not change considerably due to borrowing and lending. Here, from the simulations, we observed that the system reaches equilibrium when the number of iterations is 5 .

\section{B. Phase II: Energy Load Balancing Using Uniformly Quantized Energy Levels}

Once energy balanced clusters are formed, the cluster head within each cluster will be determined by first considering the highest quantized residual energy level followed by the one that is closest in Euclidean distance to the cluster centroid $C_{i}$. For energy load balancing using uniformly quantized energy levels, the proposed cluster head rotation procedure is explained using the following steps:

1) Once energy balanced clusters are formed the BS chooses a cluster head in each cluster that has the highest remaining quantized energy level. In case of multiple candidates, the node that is closest to the cluster centroid $C_{i}$ is chosen.

2) The BS broadcasts the ID of each cluster head along with the IDs of associated nodes to every cluster in the network. The IDs of all the associated nodes need to be send only once as the the clusters are fixed.

3) Upon receiving the frame from the BS, all nodes in the network start the sensing process.
Table I

SIMULATION PARAMETERS

\begin{tabular}{|l|l|l|}
\hline Parameter & Acronym & Value \\
\hline Initial energy & $E_{0}$ & $0.2 \mathrm{~J}$ \\
$T x$ electronics constant & $E_{\text {elec }}$ & $50 \mathrm{~nJ} / \mathrm{bit}$ \\
$R x$ electronics constant & $E_{\text {elec }}$ & $50 \mathrm{~nJ} / \mathrm{bit}$ \\
Amplifier constant & $\epsilon_{f s}$ & $10 \mathrm{pJ} / \mathrm{bit} / \mathrm{m}^{2}$ \\
Amplifier constant (multipath) & $\epsilon_{m p}$ & $0.0013 \mathrm{pJ} / \mathrm{bit} / \mathrm{m}^{4}$ \\
Data aggregation energy & $E_{D A}$ & $5 \mathrm{~nJ}$ \\
Packet size & $P$ & $64,128,256$ bytes \\
\hline
\end{tabular}

4) Associated nodes send a packet containing sensed data and their current quantized energy level to their cluster head. After each transmission, all associated nodes update their current quantized energy level.

5) Upon receiving all sensed data from the associated nodes, each cluster head aggregates the data into a single packet and sends a packet containing the aggregated sensed data to the BS. After transmission of data, each cluster head updates its current quantized energy level.

6) A list of node IDs that have energy level greater than the cluster head's current quantized energy level is also send along with aggregated data to the BS if and only if the cluster head's energy level falls below the highest energy level in the list.

7) If it is time to change the cluster head due to the energy exhaustion of any cluster head, go to Step 2.

\section{Simulation Parameters And Results}

We performed extensive MATLAB simulations to evaluate the performance of EBFC algorithm. In our simulations, the channel model and the related parameters are chosen according to the ones used in [5] and are also given in Table I. It is assumed that 100 sensor nodes each having an initial energy of $0.2 \mathrm{~J}$ are deployed randomly over a sensing field with dimensions $100 \mathrm{~m} \times 100 \mathrm{~m}$ and the BS is located at $(50,175)$.

Fig. 4 exhibits simulated lifetime curves for the proposed scheme with five clusters for various quantization levels (8, $16,32$ and 64$)$ and packet sizes (64, 128 and 256 bytes). The results are compared with dynamic cluster formation method as proposed by authors in [11] for the same packet sizes with 64 quantization levels. Since both methods use uniformly quantized energy levels for cluster head selection, the provided plots can be compared fairly to highlight the difference between fixed and dynamic clustering methods [11]. As expected, it is evident from the plots that the dynamic clustering outperforms the proposed fixed clustering method by around $14 \%$ ( 64 byte packet). Furthermore, for 128 and 256 byte sized packets the difference is around $10 \%$ and $8 \%$ respectively. Intuitively speaking, dynamic clustering was expected to perform better as the cluster formation was optimized each time when clusters were created, whereas, in fixed clustering they were only optimized initially. Hence, 


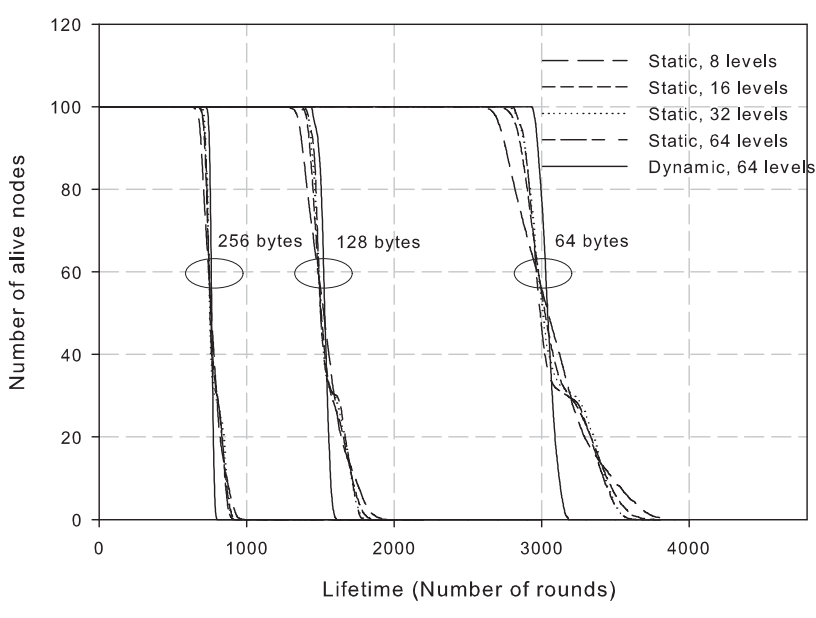

Figure 4. Lifetime curves for fixed and dynamic clustering having five clusters with $8,16,32$ and 64 uniformly quantized energy levels and 64, 128 and 256 byte packets.

the gap between the death of the first node and the last node is less (steep tail) in dynamic clustering approach than EBFC method. Despite this, the newly proposed fixed clustering algorithm has performed fairly well and it has the advantage of only one time cluster formation.

Through extensive simulations we found that the optimal number of fixed clusters is between $3-6$ which is somewhat similar to the case of dynamic clustering approach [11]. It should be noted that a few number of clusters increase intra-cluster communication overhead due to an increased distance between the sensor nodes and can result in poor performance, whereas, a large number of clusters increase the distance between the BS and the far away cluster heads which again can degrade the performance of the system. Hence, neither too few or too many clusters are expected to provide near optimal results. We also noticed that too many quantization levels also provide inferior performance due to a frequent switch in cluster heads role. Having 32 (i.e., $q=5$ ) and 64 (i.e., $q=6$ ) quantization levels provided the best results.

\section{CONCLUSION AND REMARKS}

This paper introduces a new fixed clustering algorithm (EBFC) which at first creates energy balanced clusters and then chooses a cluster head in each cluster by sharing each sensor node's energy via uniformly quantizing energy levels to apply energy load balancing and achieve a prolonged network lifetime. The results of simulations demonstrate that EBFC exhibits a fairly similar performance as its dynamic clustering counterpart due to the fact that EBFC realizes the clustering formation phase only once and has no multiple cluster formation phase related overhead.

\section{ACKNOWLEDGMENT}

This paper has been accepted for publication in the proceedings of WSN-ADT Workshop that had been held in conjunction with NTMS 2012.

\section{REFERENCES}

[1] I. F. Akyildiz, W. Su, Y. Sankarasubraminiam, and E. Cayirci, "A Survey on Sensor Networks," IEEE Communication Magazine, pp. 102-114, Vol. 40, Issue 8, August 2002.

[2] A. A. Abbasi, and M. Younis, "A survey on clustering algorithms for wireless sensor networks," Journal of Computer Communications, pp. 2826-2841, Vol. 30, Issue 14-15, October 2007.

[3] O. Boyinbode, H. Le, A. Mbogho, M. Takizawa, and R. Poliah, "A Survey on Clustering Algorithms for Wireless Sensor Networks," Proceedings of IEEE Thireenth International Conference on Network-Based Information Systems, pp. 358-364, September 2010.

[4] W. B. Heinzelman, A. P. Chandrakasan, and H. Balakrishnan, "Energy-Efficient Communications Protocol for Wireless Microsensor Networks," Proceedings of IEEE Thirty-third Hawaii International Conference on System Sciences, 2000.

[5] W. B. Heinzelman, A. P. Chandrakasan, and H. Balakrishnan, "An Application-Specific Protocol Architecture for Wireless Microsensor Networks," IEEE Transcations on Wireless Communications, Vol. 1, No. 4, pp. 660-670, October 2002.

[6] O. Younis, and S. Fahmy, "Distributed Clustering in Ad-hoc Sensor Networks:A Hybrid, Energy-Efficient Approach," Proceedings of INFOCOM. Twenty-third Annual Joint Conference of the IEEE Computer and Communications Societies, March 2004.

[7] K. Lin , H. Zhao, Y. Bi, and P. Li, "A Fixed Clustering Hierarchy Arithmetic Based on Energy Prediction for Wireless Sensor Networks," Proceedings of IEEE Fourth International Conference on Wireless Communications, Networking and Mobile Computing (WiCOM), pp. 1-5, October 2008.

[8] A. S. Zahmati, B. Abolhassani, A. A. B. Shirazi, and A. S. Bahitiari, "An Energy-Efficient Protocol with Static Clustering for Wireless Sensor Networks," International Journal of Electronics, Circuit, and Systems, Vol. 1, No. 2, pp. 135-138, May 2007.

[9] S. K. Chaurasiya, T. Pan, and S. D. Bit, "An Enhanced Energy-Efficient Protocol with Static Clustering for WSN," Proceedings of Twenty Fifth IEEE Internationl Conference on Information Networking (ICOIN), pp. 524-529, September 2008.

[10] F. Bajaber, and I. Awan, "Dynamic/Static Clustering Protocol for Wireless Sensor Network," Proceedings of IEEE Second UKSIM European Symposium on Computer Modeling and Simulation (EMS), pp. 524-529, September 2008.

[11] S. A. Ali, C. Sevgi, and A. Kocyigit, "Balancing Energy Loads in Wireless Sensor Networks through Uniformly Quantized Energy Levels-Based Clustering," Proceedings of IEEE Fourth International Conference on Sensor Technologies and Applications (SENSORCOMM), pp. 19-23, July 2010. 